\title{
Renewed considerations on the utility (or the futility) of hepatic resections for breast cancer liver metastases
}

\author{
Gian Luca Grazi \\ Hepatobiliary Biliary Pancreatic Surgery, IRCCS Regina Elena National Cancer Institute, Rome, Italy \\ Correspondence to: Gian Luca Grazi. Hepatobiliary Biliary Pancreatic Surgery, IRCCS Regina Elena National Cancer Institute, Via Elio Chianesi 53, \\ 00144, Rome, Italy. Email: gianluca.grazi@ifo.gov.it.
}

Importance: Indication for liver resection (LR) for localized hepatic metastases from breast cancer (BC) is still a matter of debate.

Objective: A literature review of recent scientific papers pertaining to hepatectomies for BC liver metastases (LM).

Evidence Review: We based our systematic review on case series on literature reviews, comparative studies and cost-utility analysis which have been selected based on criteria regarding surgery, possible prognostic factors and evaluation of long-term survival.

Findings: There is a strong inhomogeneity in the reported data, with 5 -year survivals ranging from $21 \%$ to $58 \%$. There is no agreement in the evaluation of prognostic variables predicting good survival, with the only exception of the time of treatment of the primary BC until the diagnosis of metastases. Three out of the four comparative studies report better survivals for patients who underwent a hepatectomy in comparison to those treated with chemotherapy alone, but their strength in terms of scientific evidence is weak. The only costutility analysis revealed that 2 out of the 3 scenarios considered were in favor of the treatment with surgery followed by conventional chemotherapy.

Conclusions: There is no definitive proof on the effectiveness of LRs for BC LM. Surgery can be proposed when it is possible to perform radical surgery, with R0 margins and saving at least $30 \%$ of the liver with its vascular and biliary connections. Stable skeletal metastases are not a contraindication. The interval between treatment of the primary location and diagnosis of hepatic metastases is the only prognosis criteria available.

Keywords: Breast cancer (BC); liver metastases (LM); liver surgery; hepatectomy

Submitted Apr 13, 2019. Accepted for publication Jul 12, 2019.

doi: $10.21037 / \mathrm{hbsn} .2019 .07 .12$

View this article at: http://dx.doi.org/10.21037/hbsn.2019.07.12

\section{Introduction}

Tumor of the breast is one of the most common types of cancers in women. In 2018, the estimated age-adjusted annual incidence of breast cancer (BC) in 40 European countries was $145.2 / 100,000$ in females and the mortality rate was $32.8 / 100,000$ (1).

The incidence of this tumor increased after the introduction of mammography screening and continues to grow with an ageing population. The prevalence is also on the rise due to the already cited increased incidence and to improvements in treatment outcomes.

Instead, the mortality rate has decreased in most Western countries, due to better treatment modalities and earlier detection. BC remains today the leading cause of cancerrelated deaths in European women (2). The principal cause of death is metastatic spread. The timing and the distribution of secondary tumors from $\mathrm{BC}$ has a great range of variation (3).

The routine search for tumor spreads after surgery performed on the primary location is not considered in current guidelines (4). Patients are followed by recording their history, performing physical examinations and undergoing regular mammography (4). These evaluations are performed every 3 to 6 months for the first 3 years, 
Table 1 Different considerations for hepatectomy for liver metastases from breast cancer versus those from colorectal cancer

\begin{tabular}{lll}
\hline Items & Liver metastases from BC & Liver metastases from colon cancer \\
\hline Effective chemotherapy & Established long & Established recently \\
Role of liver surgery & $\begin{array}{l}\text { Even if disease readily resectable, always treated by } \\
\text { first-line chemotherapy }\end{array}$ & Only effective first-line treatment \\
Surgical treatment & Excessively invasive & - \\
\hline
\end{tabular}

$\mathrm{BC}$, breast cancer.

every 6 to 12 months for the two subsequent years and annually thereafter. Use of complete blood counts, chemistry panels, bone scans, chest radiography, liver ultrasounds, computed tomography scans, PET scanning, magnetic resonance or tumor markers is not recommended for routine follow-up in an otherwise asymptomatic patient with no specific findings during clinical examination. Thus, a systematic search for distant metastases is usually not performed.

There are several reasons behind this clinical attitude. The main reason emerged when the first studies on the management of BCs were published. In early papers (5), different outcomes were reported according to the site of appearance of a distant metastasis. Patients developing bone metastasis had a median survival of 48 months with a strong positive effect of the first hormonal therapy administered, which was effective in $87 \%$ of patients (median response 10 months). Undergoing any kind of chemotherapy was effective in the $93 \%$ of patients (median response 11 months). On the other hand, patients which developed metastases in any other site had a significantly shorter median survival of 17 months (5).

This concept had rapidly spread and embedded itself throughout clinical practice where its influence is still evident. In addition, metastatic spread from BC has universally been defined as "systemic", thus precluding any treatment considerations in treating localized diseases.

Given that accurate data on metastatic spread could not be obtained, approximately $30 \%$ of patients with BC will develop metastases at some point during the course of the disease (6). The liver is the third most frequent site of metastatic spread (after lymph nodes and lung). Only 5-25\% of patients will have isolated BC liver metastases (LM). These patients could potentially be eligible for hepatic surgery or other liver-directed therapies (6). The number of patients with isolated LM is thus a substantial amount.

In spite of this large number of patients, the considerations on possible treatment has always been considered marginal. There are different considerations for isolated LM from BC in comparison to those for isolated LM from colorectal cancers (7). Table 1 summarizes these differences. Chemotherapy treatment has shown to be effective in isolated metastases from BC that was established a long time ago and is usually employed as first line therapy. Furthermore, surgical treatment for the liver is perceived to be excessively invasive, especially after BC management which has constantly evolved toward a more conservative approach. Instead, surgical therapy is the preferred first-line treatment for metastases from colorectal cancer.

A collective series from 8 international centers during a 24 year-period, collected only 119 cases of liver resections (LRs) performed for BC LM (6). Assuming that approximately $10 \%$ of women with $\mathrm{BC}$ will develop metastatic disease confined to the liver, it can be postulated that in the United States only 96,000 cases may arise over the same period (8). Even if we consider this estimation, there is a large discrepancy between the theoretical number of potential candidates for liver-directed treatments and the number of LRs effectively performed.

This is in stark contrast with what the worldwide development of specialized liver centers states. The increased safety of LRs led to expand indications for surgery to a large variety of LM. There are at least two studies that reported this expansion of the activity for non-colorectal non-endocrine LM both in Europe and in the United States $(9,10)$. In the French report, LR for BC metastases represent one third of all the procedures, with a median survival of 45 months (9) and cumulative survivals and recurrence-free survivals comparable with those obtained for all the other resections for non-colorectal non-endocrine LM. Surgery performed for LM from BC carried a "protective" effect in a well-known clinical score employed to stratified patient survival after hepatectomy (9).

In spite of these already published data, which suggested the feasibility and the safety of LR in this setting, we still do not have clear evidence of their real efficacy in terms of 
Table 2 More recent literature reviews appeared over the past 10 years

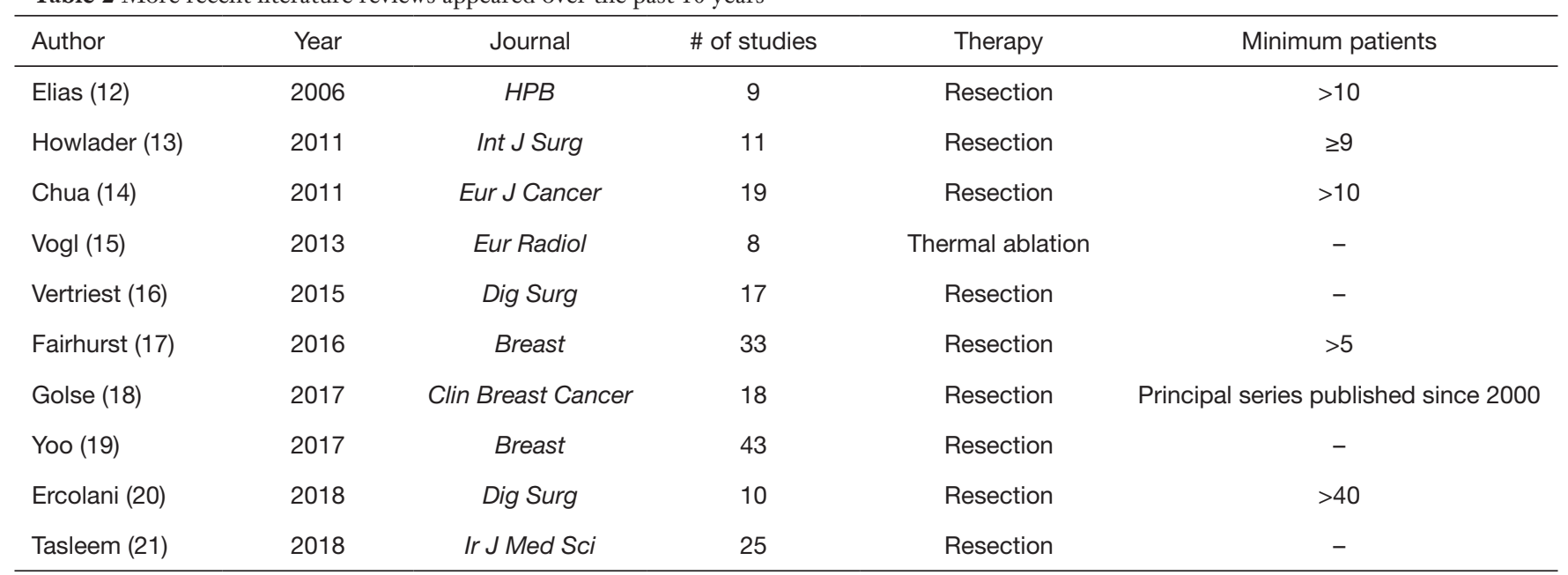

improving patient survival. Most of the already cited papers are from single center reviews, that are retrospective and usually lack defined selection criteria for surgery. In other words, the proof of the usefulness of surgery for these patients is still the main issue in question (11), which has remained unanswered for over the past 30 years. I present the following article in accordance with the Narrative Review reporting checklist (available at https://hbsn. amegroups.com/article/view/10.21037/hbsn.2019.07.12/rc).

\section{Literature review}

One possible way to overcome the lack of consistent data is to collect those already present in the literature and carry out a comprehensive analysis of them. There are several manuscripts that focus on this goal as the main purpose. However, even these reports contain significant and unresolved problems.

If we consider only the more recent reviews published, we obtain 10 papers from 2006 to 2018 (Table 2). They all include series on LRs except for one, which reported on percutaneous thermal ablation (15). The selection criteria differ from paper to paper, with the number of studies included in a single review varying from 8 to 43 . Several papers included only studies with a defined number of surgical procedures performed. Obviously, the more the number of procedures selected increases, the more the number of considered papers decreases. Only one paper selected a series with more than 40 cases, resulting in only 10 series available (20). Two reviews included a series with at least 10 cases, including 9 and 19 series, respectively
$(12,14)$. One paper included the "principal series published since 2000", collecting 18 papers (18).

The first obvious problem is the inhomogeneity of the reported data, with postoperative mortality rates ranging from $0 \%$ to $5 \%$ and 5 -year overall survivals ranging from $21 \%$ to $58 \%$, thus introducing questions regarding patient selection (Table 3).

The second aspect involves the heterogeneity of the variables considered in evaluating the prognostic factors after LR. The tables published reflect the different approaches that were adopted towards treating the disease, where the final results did not give the reader indications for patient selection.

Last, but not least, due to the scarcity of procedures performed for this indication, the study periods are usually very long, in order to collect a critical number of cases to run acceptable evaluations, thus introducing potential bias in terms of selection, staging and surgical technique.

The largest review was published recently in a non-surgical journal (19). This paper takes into account 43 papers that met defined search criteria, from a pool of 1,705 potentially relevant citations identified. The reported 30-day mortality rate is $0.7 \%$ (6 patients out of the 918 were included in studies where this data was available). Median 3-and 5-year overall survivals were $56 \%$ and $37 \%$, respectively (Table 3 ).

Only $21(48.8 \%)$ out of 43 studies reported some selection criteria for surgery. Only $6(14.0 \%)$ studies included more than 50 patients.

Many studies were inconsistent concerning the variables included for identifying possible prognostic factors. Only 22 $(51.2 \%)$ out of the 43 papers reported such an investigation. 


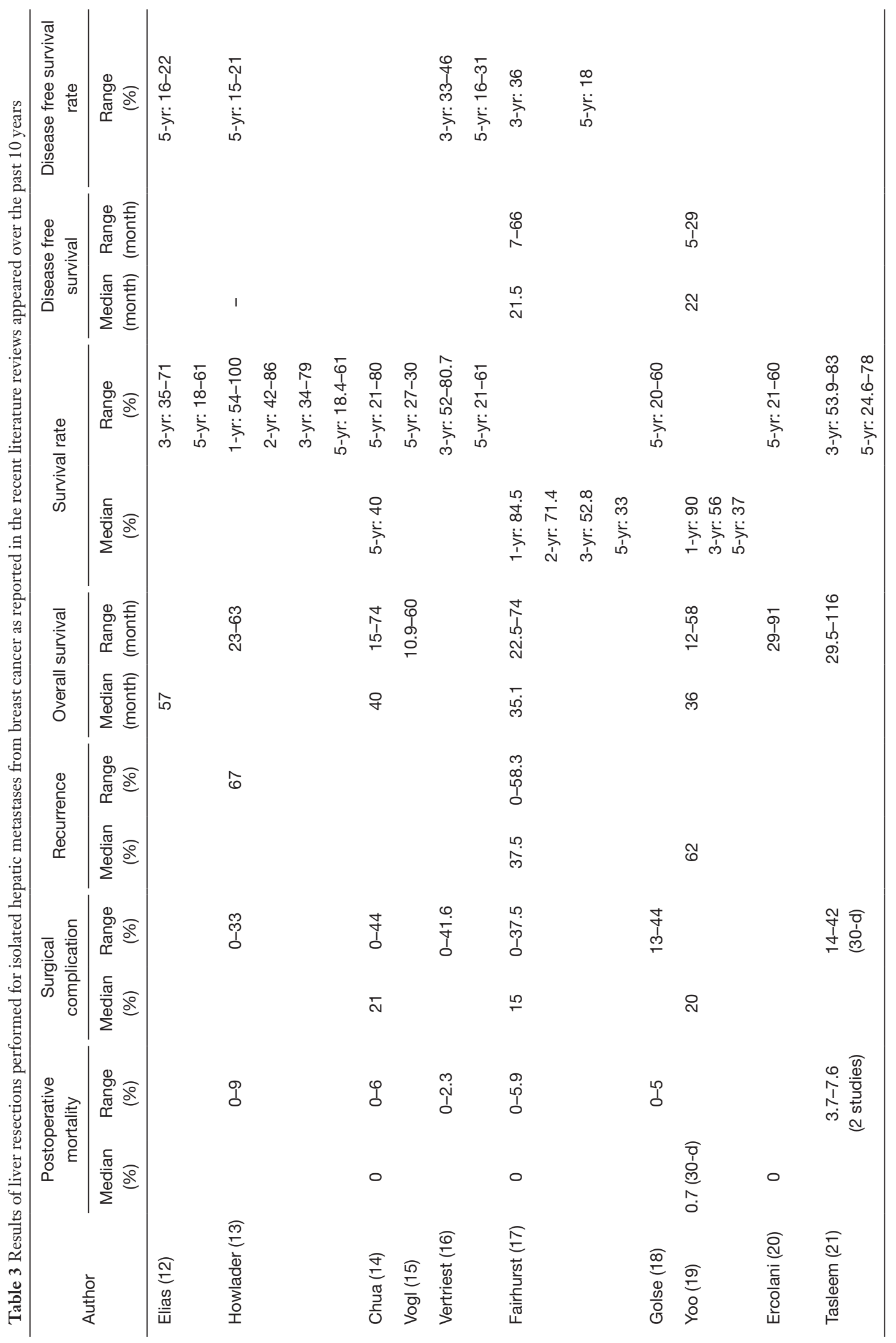


Many of the variables were reported in some manuscripts but not in others. In other cases, the variables had different meanings from paper to paper. Among the many, the simple variable "age" was reported only in 15 (68.2\%) of these 22 studies. In 12 studies, this variable had no influence on long-term results; in 2 studies better results were reported in patients over 50 years of age $(22,23)$; in one study survival was better in patients younger than 50 (24).

One single factor that was shown to have some positive influence on survival was the prolonged interval between BC treatment and the diagnosis of LM (19).

In conclusion, the analysis of a series that included a few clinical cases covered significant heterogeneity in terms of selection criteria, staging of the disease and treatment characteristics.

Apart from the confusion created by the different reviews, the paper by Ruiz et al., reports the experience of a single center with 162 hepatectomies performed in 139 consecutive women (25). The paper reports on negative factors predicting survival which include: (I) more than 1 LM; (II) being "triple negative" (negative receptor status for estrogen, progesterone and HER2/Neu receptor); (III) microscopic vascular invasion. On the other hand, (I) negative resection margins; (II) administration of hormonal therapy before and after LR; (III) the performance of repeated hepatectomy were all positive predictors of longterm survival.

This is the only paper that provides the reader with a nomogram to predict 3-and 5-year survival after hepatectomy. In terms of patient selection, it would be substantially better to be able to prognosticate with all data available before surgery rather than after.

\section{Comparative studies}

The strongest bodies of evidence in terms of therapy efficacy come from comparative studies. There are several ways to obtain comparative evaluations with the aid of more or less sophisticated statistical tools when it is not possible to perform randomized, prospective, controlled studies. Today, in the field of liver-directed therapies for localized LM from BC, it is impossible to find strong comparative studies and their consideration in terms of scientific strength is poor.

The first paper emerged from France (7). It is a retrospective evaluation of 51 patients (out of 100 selected and available) medically treated and taken from a multidisciplinary staff conference database. They were compared with 49 patients (out of 100 patients treated with surgery) matched for age at $\mathrm{BC}$ diagnosis, year of $\mathrm{BC}$ diagnosis, time between $\mathrm{BC}$ diagnosis and first LM, TNM stage of BC, hormone receptor status, ductal or lobular histology. The selection criteria adopted by this paper for surgery were (I) resectable LMs ( $\leq 4$ metastases); (II) stable disease or disease responding to chemotherapy and/ or hormone therapy; (III) performance status (PS) of 0-1. The only extrahepatic site of metastases allowed was bone metastases if they did not grow during treatment.

They were able to demonstrate a significant better survival in the surgical treated group. Better predictors of survival for the whole cohort of 100 patients were: (I) negative lymph node status of the primary tumor; (II) received a single course of chemotherapy; (III) no bone metastases at the time of LM; (IV) underwent liver surgery.

The second paper appeared in the same year and included 38 patients treated with chemotherapy over a 7 -year period compared to 26 patients with isolated LM that received liver-directed therapies (26). Twelve had LRs when the disease was confined to one lobe regardless of the number of lesions; 14 had percutaneous radiofrequency ablation if the metastases had a bilobar distribution and there were no lesions greater than 6 centimeters (26). They were able to demonstrate prolonged survival in treated patients in comparison to the control group (median survival 21.1 versus 9.7 months) with patients treated by LR performing better than those treated by local ablation.

A third paper was published in 2016 (27), that included 167 patients out of the 2,150 in a 23 -year period that were evaluated by a multidisciplinary tumor board. Patients treated with LR and/or ablation (surgical cohort: 69 patients, $41 \%$ ) and those receiving medical therapy alone (medical cohort: 98 patients, 59\%) were analyzed and compared. A propensity score analysis was used to control for selection bias, resulting in an uneven distribution of covariates among groups.

This paper did not show any benefits in the performance of liver-directed therapies in comparison to medical therapy alone, but the authors did claim that surgical therapy played a role in providing at least a temporary free-of-disease status to patients, who had a chance to discontinue toxic therapy at least for some time.

The last paper reports on the activity of a multidisciplinary tumor board in a tertiary referral center; thus, its comparative nature is less powerful than the previous papers (28).

They reported on 61 patients with isolated LM that were treated with surgery (23 patients), percutaneous 
Table 4 Summary of the comparative studies on therapy for liver metastases from breast cancer appeared in the literature up to now

\begin{tabular}{|c|c|c|c|c|}
\hline & Mariani (7) & Polistina (26) & Sadot (27) & Abbas (28) \\
\hline Nature of the study & Retrospective & Retrospective & Retrospective & $\begin{array}{l}\text { Retrospective (report of UK } \\
\text { tertiary center tumor board } \\
\text { meeting) }\end{array}$ \\
\hline Medical cohort & Medical therapy alone & Medical therapy alone & Medical therapy alone & Medical therapy alone \\
\hline \multirow{2}{*}{$\begin{array}{l}\text { Inclusion criteria for } \\
\text { medical cohort }\end{array}$} & With/without bone mets & \multirow{2}{*}{ Not reported } & \multirow{2}{*}{ Not reported } & \multirow{2}{*}{ Not reported } \\
\hline & No other mets & & & \\
\hline Surgical cohort & Resection only & Resection or ablation & Resection and/or ablation & Resection and/or ablation \\
\hline $\begin{array}{l}\text { Inclusion criteria for } \\
\text { surgical cohort }\end{array}$ & PS 0-1 & No underlying CLD & Non reported & Resectable \\
\hline Statistics & Case control study & Analysis of survival & $\begin{array}{l}\text { Propensity score analysis } \\
\text { (Mariani and Polistina's } \\
\text { studies not cited) }\end{array}$ & $\begin{array}{l}\text { Analysis of survival } \\
\text { (Mariani and Polistina's } \\
\text { studies not cited) }\end{array}$ \\
\hline
\end{tabular}

Mets, metastases; PS, performance status; CLD, chronic liver disease.

radiofrequency ablation (11 patients) and chemotherapy alone (27 patients). LR or ablation were proposed to patients when the disease was considered resectable at first evaluation or when the disease responded to systemic chemotherapy and then considered resectable. A better survival was reported when patients had undergone surgery or ablation. The presence of extrahepatic disease considerably reduced survival after any treatment.

Table 4 summarizes and compares these considerations.

\section{Cost utility analysis}

There is only one cost-utility analysis published (29). This paper takes into consideration 2 different scenarios with 3 different strategies. In both scenarios, strategy A is represented by the performance of a LR followed by postoperative conventional systemic therapy. In the first scenario, strategy A is compared with conventional systemic chemotherapy alone (strategy B). In the second scenario, strategy A is compared with a newer systemic therapy alone (strategy C).

LR plus systemic therapy was more cost-effective for patients with ER+ tumors than systemic therapy alone.
Instead, for patients with HER2+ tumors, the performance of a hepatectomy plus systemic chemotherapy had a costeffectiveness that is comparable to systemic therapy alone. Furthermore, LR plus standard systemic chemotherapy was more cost-effective when compared with the administration of newer systemic chemotherapeutic agents.

The main prognostic factors associated with patient survival are those related to the biologic behavior of the tumor. The diagnosis of an ER- primary tumor and a preoperative disease progression were both associated with a poor outcome after hepatic resection. On the other hand, among patients with ER+ tumors who had stable or responsive disease at systemic therapy, surgical therapy was strongly associated with an improved survival compared with chemotherapy alone.

\section{Discussion}

LRs conducted for LM from BC represent an unresolved question for liver surgeons. The present paper attempts to analyze the already available scientific literature regarding this topic. Up to now, the vast majority of papers published include single center reports, including more or less 
extensive reviews on what is already available.

Recent literature reviews, comparative studies and the only cost utility analysis have thus been taken into consideration in the attempt to verify the presence of certainty on the argument. In the end, it must be stated that there are no strong lines of evidence for including surgical therapy within the advised treatments for LM from BC. Present guidelines state that the first approach for metastatic diseases is to undergo systemic chemotherapy that is what currently takes place in clinical practice.

There seems to be no scientific literature supporting any particular feature of LM from BC may be assumed as a determinant to be employed as a criterion for surgery (18). There is a great heterogeneity in terms of the clinical variables considered, number of patients included, time of observation, etc., reported in these papers. The conclusions of these reviews are quite confounding and not useful for providing advice on who should be operated or not. The question raises the opportunity to continue to publish such papers in the future.

Most of these papers conclude that LR is an effective treatment for patients carrying LM from BC. This is effectively true for some highly selected patients. However, we must consider that the progress achieved in the field of liver surgery in terms of safety are well-known, with a post-operative mortality rate in patients with normal liver or after chemotherapy already being very low, often less than $1 \%$.

The fact that LR is really an effective treatment for these patients should be supported by robust comparative studies on long-term survival, which are not currently available. It is therefore difficult to accept the conclusions of these papers with the enthusiasm that often transpires. It is impossible to state what the survival rate of these patients would have been if treated with chemotherapy alone and knowing that in some cases the disease has an indolent course indeed. It is unlikely that a comparative, prospective single center trial will be ever feasible.

Even if they should not be included in the category "reviews", the two papers from Paul Brousse's group do deserve attention $(9,25)$. Their policy is quite aggressive but they are the only publishing series of repeated LRs for hepatic recurrence. A particular effort was made by setting up a nomogram to predict the outcome of patients after LR. But some of the variables included in this nomogram are detectable only after surgery and therefore the nomogram itself is not useful in providing indications before performing a hepatectomy.
There are four comparative studies available. Their designs are rather weak; thus, the strength of the evidence they support is poor. All of them are retrospective. One is a case-control study (7) and the second is selected according to the propensity score analysis (27). Only one (7) reports the inclusion criteria in the medical arm as a control group. Only one paper includes LR alone (7) while the other combined with patients treated with percutaneous ablation. Two papers describe their inclusion criteria for surgery. Three papers $(7,27,28)$ obtained their data from a multidisciplinary tumour board meeting.

Three out of the four papers $(7,26,28)$ reports favorable results in patients treated with LR or thermal ablation when compared to those treated with chemotherapy alone.

Surprisingly, when indications for surgery are reported, the presence of multiple nodules does not represent a contraindication. Mariani carried out the surgical procedure in the presence of 4 or less metastases and Polistina performed a hepatectomy in the presence of LMs confined to one lobe regardless of the number.

Adding these considerations to those reported by the Paul Brousse's group (25), it seems that indications for surgery can be compared with those applied for colorectal LMs, where surgery is performed when the removal of the disease is possible, leaving a $30 \%$ of healthy liver parenchyma, without injuring vascular inflow nor outflow and biliary drainage, obtaining a R0 operation. Stable disease seems to be the preferred option, with the longest interval between BC treatment and diagnosis of LM being the only possible preoperative favorable prognostic factor. The presence of stable skeletal metastases did not emerge as an absolute contraindication. None of the centers has described using a preoperative downstaging strategy with chemotherapy, nor did they report patients becoming resectable from a non-operable situation, thus confirming that the indications for LR are often extemporary.

The only paper that did not demonstrate any surgical advantage over medical treatment claimed that surgery could at least decrease the need for repetitive cycles of cytotoxic chemotherapy, and could reduce the tumor burden providing an immunologic benefit, thus allowing time off cytotoxic chemotherapy during the disease-free period (treatment-free holiday) (27). To our knowledge, this is the only paper reporting such considerations and it is thus hard to justify indications for LR based on this.

It is quite evident that a large number of metastatic patients from $\mathrm{BC}$ are still being managed medically where only very few of them have been discussed during 
multidisciplinary tumor board teams that included a liver surgeon. When this occurs, LR is considered a safe and viable option in the therapeutic scenario. One must keep in mind that the three scientific studies reporting on the activities of multidisciplinary tumour groups evaluating LM from BC came to different conclusions $(7,27,28)$.

The only cost-utility analyses performed using the Monte-Carlo simulation revealed that two out of three scenarios were in favor of the use of LR plus conventional systemic therapy (29). Selection criteria for surgery were obtained by a single case-control study (7), which already demonstrated better results after hepatectomy in comparison to with medical therapy. The paper's influence on clinical activity is unpredictable as it has been published in a surgical journal that may not be distributed among Colleagues that specifically deal with $\mathrm{BC}$ within specialized units.

Today, metastatic diseases from $\mathrm{BC}$ remains within the management of the oncologist limiting the chances for hepatic surgeons to ever have a true role in the management of these patients. All around the world there are several breast units that effectively treat the large number of BC patients with definite and approved diagnostic and therapeutic pathways; these guidelines do not consider liver surgery in cases of isolated LM.

Given the minimal involvement of our colleague Oncologists and the lack of randomized, prospective studies that could greatly influence scientific evidence, it will be very difficult for these patients to receive alternative treatment to chemotherapy.

The task force on BC metastases of the European School of Oncology in 2007 (30) stated that "A small but very important subset of metastatic BC patients, for example those with a solitary metastatic lesion, can achieve complete remission and a long-term survival. For these selected patients, a more aggressive and multidisciplinary approach should be considered". Up to now, nothing has changed in this field and hepatic surgeons still continue to sporadically remove LM from the liver from patients that, due to the biological feature of their disease, will probably have the same long term survival without surgery.

One should keep in mind that several papers recommend that the selection for possible surgery should be performed only in tertiary referral centers. In these centers, the best short-term (low morbidity, absent postoperative mortality) and long-term outcomes can be offered to patients regarding liver surgery. The fact that the centralization of surgery, and in particular of the oncological surgery, thus including hepato-biliary-pancreatic surgery, entails several advantages in terms of results for patients is now a well-established fact (31). However, the consideration made above must be repeated. While medical oncology is a very common specialized unit even in peripheral hospitals, oncological surgery is much less so. In the absence of structured oncological networks that can be used as a reference for the evaluation and treatment of these patients, it still is difficult to think that patients with liver-isolated metastases can easily access such complex clinical evaluations. This certainly represents an additional complication for applying LR in patients that may benefit from them.

In conclusion, even if many surgical papers reported achieving excellent results with LR for BM in terms of improved survival, it must be stated that there are no scientific certainties to absolutely validate these considerations. Indications for performing LR in these patients should not differ from those that are applied in surgery for colorectal LM: performing radical surgery with R0 margins and saving at least $30 \%$ of the liver with its vascular and biliary connections. Stable bone metastases are not a contraindication for surgery. The free interval between the treatment of the primary tumor and the diagnosis of LM is the only prognosis criteria that has been defined in these patients.

\section{Acknowledgments}

Funding: None.

\section{Footnote}

Reporting Checklist: The author has completed the Narrative Review reporting checklist. Available at https://hbsn. amegroups.com/article/view/10.21037/hbsn.2019.07.12/rc

Conflicts of Interest: The author has completed the ICMJE uniform disclosure form (available at https://hbsn. amegroups.com/article/view/10.21037/hbsn.2019.07.12/coif). The author has no conflicts of interest to declare.

Ethical Statement: The author is accountable for all aspects of the work in ensuring that questions related to the accuracy or integrity of any part of the work are appropriately investigated and resolved.

Open Access Statement: This is an Open Access article distributed in accordance with the Creative Commons 
Attribution-NonCommercial-NoDerivs 4.0 International License (CC BY-NC-ND 4.0), which permits the noncommercial replication and distribution of the article with the strict proviso that no changes or edits are made and the original work is properly cited (including links to both the formal publication through the relevant DOI and the license). See: https://creativecommons.org/licenses/by-nc-nd/4.0/.

\section{References}

1. ECIS-European Cancer Information System. Estimates of cancer incidence and mortality in 2018, for all cancer sites. Accessed 2019, January 28. Available online: https:// ecis.jrc.ec.europa.eu/explorer.php?\$0-0\$1-AE28\$2-All\$41\$3-All\$6-0,14\$5-2008,2008\$7-7\$CEstByCancer\$X0_83\$CEstRelativeCanc\$X1_8-3\$X1_9-AE28

2. Senkus E, Kyriakides S, Ohno S, et al. Primary BC: ESMO Clinical Practice Guidelines for diagnosis, treatment and follow-up. Ann Oncol 2015;26 Suppl 5:v8-30.

3. Schwartz RS, Erban JK. Timing of Metastasis in BC. N Engl J Med 2017;376:2486-8.

4. Khatcheressian JL, Hurley P, Bantug E, et al. BC followup and management after primary treatment: American Society of Clinical Oncology clinical practice guideline update. J Clin Oncol 2013;31:961-5.

5. Sherry MM, Greco FA, Johnson DH, et al. Metastatic BC confined to the skeletal system. An indolent disease. Am J Med 1986;81:381-6.

6. Margonis GA, Buettner S, Sasaki K, et al. The role of liver-directed surgery in patients with hepatic metastasis from primary BC: a multi-institutional analysis. HPB (Oxford) 2016;18:700-5.

7. Mariani P, Servois V, De Rycke Y, et al. LM from BC: Surgical resection or not? A case-matched control study in highly selected patients. Eur J Surg Oncol 2013;39:1377-83.

8. D'Angelica M. Hepatic resection for metastatic BC: an exercise in selection bias. HPB (Oxford) 2016;18:631-2.

9. Adam R, Chiche L, Aloia T, et al. Hepatic resection for noncolorectal nonendocrine LM: analysis of 1,452 patients and development of a prognostic model. Ann Surg 2006;244:524-35.

10. Groeschl RT, Nachmany I, Steel JL, et al. Hepatectomy for noncolorectal non-neuroendocrine metastatic cancer: a multi-institutional analysis. J Am Coll Surg 2012;214:769-77.

11. Elias D, Lasser P, Spielmann M, et al. Surgical and chemotherapeutic treatment of hepatic metastases from carcinoma of the breast. Surg Gynecol Obstet $1991 ; 172: 461-4$.
12. Elias D, Di Pietroantonio D. Surgery for LM from BC. HPB (Oxford) 2006;8:97-9.

13. Howlader M, Heaton N, Rela M. Resection of LM from BC: towards a management guideline. Int J Surg 2011;9:285-91.

14. Chua TC, Saxena A, Liauw W, et al. Hepatic resection for metastatic BC: a systematic review. Eur J Cancer 2011;47:2282-90.

15. Vogl TJ, Farshid P, Naguib NN, et al. Thermal ablation therapies in patients with BC LM: a review. Eur Radiol 2013;23:797-804.

16. Vertriest C, Berardi G, Tomassini F, et al. Resection of single metachronous LM from BC stage I-II yield excellent overall and disease-free survival. Single center experience and review of the literature. Dig Surg 2015;32:52-9.

17. Fairhurst K, Leopardi L, Satyadas T, et al. The safety and effectiveness of LR for BC LM: A systematic review. Breast 2016;30:175-84.

18. Golse N, Adam R. Liver Metastases From Breast Cancer: What Role for Surgery? Indications and Results. Clin Breast Cancer 2017;17:256-65.

19. Yoo TG, Cranshaw I, Broom R, et al. Systematic review of early and long-term outcome of LR for metastatic BC: Is there a survival benefit? Breast 2017;32:162-72.

20. Ercolani G, Zanello M, Serenari M, et al. Ten-Year Survival after LR for Breast Metastases: A Single-Center Experience. Dig Surg 2018;35:372-80.

21. Tasleem S, Bolger JC, Kelly ME, et al. The role of LR in patients with metastatic BC: a systematic review examining the survival impact. Ir J Med Sci 2018;187:1009-20.

22. Lubrano J, Roman H, Tarrab S, et al. Liver resection for breast cancer metastasis: does it improve survival? Surg Today 2008;38:293-9.

23. Treska V, Cerna M, Liska V, et al. Surgery for breast cancer liver metastases - factors determining results. Anticancer Res 2014;34:1281-6.

24. Dittmar Y, Altendorf-Hofmann A, Schule S, et al. Liver resection in selected patients with metastatic breast cancer: a single-centre analysis and review of literature. J Cancer Res Clin Oncol 2013;139:1317-25.

25. Ruiz A, Wicherts DA, Sebagh M, et al. Predictive ProfileNomogram for LR for BC Metastases: An Aggressive Approach with Promising Results. Ann Surg Oncol 2017;24:535-45.

26. Polistina F, Costantin G, Febbraro A, et al. Aggressive treatment for hepatic metastases from BC: results from a single center. World J Surg 2013;37:1322-32.

27. Sadot E, Lee SY, Sofocleous CT, et al. Hepatic Resection 
or Ablation for Isolated BC LM: A Case-control Study With Comparison to Medically Treated Patients. Ann Surg 2016;264:147-54.

28. Abbas H, Erridge S, Sodergren MH, et al. BC LM in a UK tertiary centre: Outcomes following referral to tumour board meeting. Int J Surg 2017;44:152-9.

29. Spolverato G, Vitale A, Bagante F, et al. LR for BC LM: A
Cost-utility Analysis. Ann Surg 2017;265:792-9.

30. European School of Oncology (ESO)-MBC Task Force. Metastatic breast cancer. Recommendations proposal from the European School of Oncology (ESO)-MBC Task Force. Breast 2007;16:9-10.

31. Vonlanthen R, Lodge P, Barkun JS, et al. Toward a Consensus on Centralization in Surgery. Ann Surg 2018;268:712-24.
Cite this article as: Grazi GL. Renewed considerations on the utility (or the futility) of hepatic resections for breast cancer liver metastases. HepatoBiliary Surg Nutr 2021;10(1):49-58. doi: $10.21037 /$ hbsn.2019.07.12 